\title{
ON A GENERALIZED TZITZEICA EQUATION
}

\author{
RALPH WILLOX \\ Graduate School of Mathematical Sciences, The University of Tokyo, 3-8-1 Komaba, Meguro-ku, \\ 153-8914 Tokyo, Japan \\ e-mail:willox@poisson.ms.u-tokyo.ac.jp
}

(Received 26 November, 2003; accepted 11 February, 2004)

\begin{abstract}
Extending the so called coupled KP hierarchy to negative time flows one obtains coupled Toda-type equations defined on a two-dimensional lattice. These equations allow for reductions to $1+1$ dimensional integrable systems that are defined on a finite part of this lattice. A system of coupled Hirota bilinear equations, obtained from such a reduction and defined on only 5 points of the lattice, will be shown to correspond to a coupling of a Tzitzeica equation to two linear equations. The Lax representation of this system is also presented.
\end{abstract}

2000 Mathematics Subject Classification. 37K10, 35Q58.

1. Introduction. Together with the famous sine-Gordon equation, the Tzitzeica equation [23]

$$
(\log \mathcal{H})_{X Y}=\mathcal{H}-\frac{1}{\mathcal{H}^{2}}
$$

is one of the prime examples of integrable systems that appear in classical differential geometry (see e.g. [7] for a recent survey of the interrelations that exist between research in integrable systems and geometry). As an integrable equation it has been "discovered" and re-discovered at various times by a large number of authors, cf. for example [3, $\mathbf{6}, \mathbf{1 1}, \mathbf{1 2}, \mathbf{1 9}$ ]. Before proceeding with a dicussion of an integrable generalization of this equation to a coupled system, it is worthwhile to look into the origins of the Tzitzeica equation itself within a larger integrable framework; i.e. in the context of the 2-dimensional (2D) Toda lattice. The reader is referred to the introductory part of [22] for an excellent account of the Tzitzeica equation (1) within its classical context of hyperbolic surfaces in centro-affine geometry.

1.1. The 2D-Toda lattice and its reductions. The $2 \mathrm{D}$-Toda lattice can be expressed in terms of the Cartan matrix $\left(a_{n m}\right)$ of $A_{\infty}$ (the A-type infinite rank Kac-Moody algebra) [13]:

$$
\begin{aligned}
\forall n \in \mathbb{Z}: & \theta_{n, x y}=-\sum_{m \in \mathbb{Z}} a_{m n} \exp \left(-\theta_{n}\right), \\
a_{n m} & =\left\{\begin{array}{cl}
2 & m=n \\
-1 & m=n \pm 1 \\
0 & m \neq n, n \pm 1
\end{array}\right.
\end{aligned}
$$


It is well known that the 2D-Toda lattice can be cast into Hirota bilinear form by means of the dependent variable transformation

$$
\theta_{n}=\log \frac{\tau_{n}^{2}}{\tau_{n-1} \tau_{n+1}}
$$

yielding the following lattice equation for the tau functions $\tau_{n}$ :

$$
\frac{1}{2} D_{x} D_{y} \tau_{n} \cdot \tau_{n}=\tau_{n}^{2}-\tau_{n-1} \tau_{n+1} \quad(n \in \mathbb{Z})
$$

The 2D-Toda lattice (2) can be obtained from the compatibility condition of a discrete Zakharov-Shabat-type system $\left(v_{n}=\partial_{x} \log \frac{\tau_{n-1}}{\tau_{n}}\right)$

$$
\left\{\begin{array}{l}
\phi_{n, x}=v_{n} \phi_{n}+\phi_{n-1} \\
\phi_{n, y}=\exp \left(-\theta_{n}\right) \phi_{n+1}
\end{array} \quad(n \in \mathbb{Z})\right.
$$

and as such, it can be shown to be integrable through the inverse scattering method [19]. The tau functions that appear in (4) can be expressed as Grammian determinants involving solutions of this linear system and its (formal) adjoint [21].

The 2D-Toda lattice contains a wealth of integrable (sub-)systems which can be obtained from it by restricting the infinite lattice (2) to a semi-infinite one (e.g., one labeled by an index $n \in \mathbb{N}$ ) or to a finite lattice. This can be achieved by one of the following reduction procedures (or by a combination of them).

(i) Periodic reductions. Imposing $\tau_{n+p}=\tau_{n}$ (for some $p \in \mathbb{N}$ ) on the lattice (4) reduces the 2DToda lattice to a finite lattice that will correspond to the Cartan matrix of the affine Lie algebra $A_{p-1}^{(1)}$ (in the sense of formula (2)). The linear system that underlies the resulting lattice is obtained from (5) by requiring $\phi_{n}=\lambda^{-n} \phi_{[n]}([n]:=n \bmod p)$ for some spectral parameter $\lambda \in \mathbb{C}$. As an example one may cite the hyperbolic sineGordon (sinh-Gordon) equation which is obtained at $p=2$.

(ii) $\mathbf{B}$ and $\mathbf{C}$ type reductions. A reduction of (2) to a semi-infinite lattice can be achieved by imposing one of two possible constraints:

$$
\begin{array}{ll}
\text { B-type: } & \tau_{n}=\tau_{1-n} \\
\text { C-type: } & \tau_{n}=\tau_{-n}
\end{array}
$$

i.e., by "folding" the tau function lattice (4) in between the tau functions $\tau_{0}$ and $\tau_{1}$, resulting in a Toda lattice that corresponds to the Cartan matrix for $B_{\infty}$, or exactly on the tau function $\tau_{0}$ which yields a Toda lattice associated to the Cartan matrix for $C_{\infty}$. Imposing periodicity as in the case of reduction (i) on these lattices reduces them to finite Toda lattices that correspond to affine Lie algebras $A_{2 r}^{(2)}, D_{r}^{(2)}$ or $C_{r}^{(1)}$, depending on the parity of the period $p$ (see e.g. [21] for further details concerning these reductions and the algebras associated to them). The Tzitzeica equation (1) can be seen to correspond to a period 3 B-type Toda lattice. 
1.2. The Tzitzeica equation. Imposing a B-type reduction (6) on (4) yields a semi-infinite lattice of tau functions

$$
\begin{aligned}
\frac{1}{2} D_{x} D_{y} \tau_{0} \cdot \tau_{0} & =\tau_{0}^{2}-\tau_{0} \tau_{-1} \\
\frac{1}{2} D_{x} D_{y} \tau_{-1} \cdot \tau_{-1} & =\tau_{-1}^{2}-\tau_{0} \tau_{-2}
\end{aligned}
$$

which corresponds to a $B_{\infty}$-type Toda lattice through the dependent variable transformation

$$
\theta_{0}=\log \frac{\tau_{0}}{\tau_{-1}}, \quad \theta_{-n}=\log \frac{\tau_{-n}^{2}}{\tau_{1-n} \tau_{-1-n}} \quad\left(\forall n \in \mathbb{N}_{0}\right) .
$$

An important remark at this point is that only very special $\tau_{0}$ will be compatible with this folding of the tau function lattice. More precisely, the tau functions $\tau_{0}$ that form the starting point of the $B_{\infty}$-Toda lattice are all perfect squares; i.e. they can be represented as $\tau_{0}=\tau_{\mathrm{Pf}}^{2}$ where $\tau_{\mathrm{Pf}}$ is a Pfaffian (the reader is again referred to [21] for further details).

If one now performs a 3-reduction on this B-type Toda lattice, imposing $\tau_{n+3}=\tau_{n}$, one obtains a finite sub-lattice of (8)

$$
\left\{\begin{array}{l}
\frac{1}{2} D_{x} D_{y} \tau_{0} \cdot \tau_{0}=\tau_{0}^{2}-\tau_{0} \tau_{-1} \\
\frac{1}{2} D_{x} D_{y} \tau_{-1} \cdot \tau_{-1}=\tau_{-1}^{2}-\tau_{0}^{2}
\end{array}\right.
$$

where one has to take into account the relationship $\tau_{-2}=\tau_{0}$ induced by the periodicity of the lattice $\left(\tau_{-2} \equiv \tau_{1}\right)$ and the B-type reduction that lies at its origin: $\tau_{1} \equiv \tau_{0}$. Hence

$$
\theta_{0}=\log \frac{\tau_{0}}{\tau_{-1}}, \quad \theta_{-1}=\log \frac{\tau_{-1}^{2}}{\tau_{0}^{2}} \equiv-2 \theta_{0},
$$

transforms (10) into the single nonlinear equation

$$
\theta_{0, x y}=\exp \left(2 \theta_{0}\right)-\exp \left(-\theta_{0}\right)
$$

This equation is nothing but the Tzitzeica equation (1) in its exponentiated form $\left(\mathcal{H}=\exp \left(-\theta_{0}\right)\right)$. It can also be expressed in terms of the Cartan matrix for the affine Lie algebra $A_{2}^{(2)}$ :

$$
\left(\begin{array}{c}
\theta_{-1} \\
\theta_{0}+\log 2
\end{array}\right)_{x y}=-\left(\begin{array}{cc}
2 & -4 \\
-1 & 2
\end{array}\right)\left(\begin{array}{c}
\exp \left(-\theta_{-1}\right) \\
\exp \left(-\theta_{0}-\log 2\right)
\end{array}\right) .
$$

Implementing the reductions above on the linear system (5) one obtains a Lax pair for the Tzitzeica equation $[\mathbf{2 0}]\left(v_{0}=(\log \mathcal{H})_{x}\right)$ :

$$
\left(\begin{array}{l}
\phi_{1} \\
\phi_{2} \\
\phi_{3}
\end{array}\right)_{x}=\left(\begin{array}{ccc}
0 & 0 & \lambda \\
\lambda & -v_{0} & 0 \\
0 & \lambda & v_{0}
\end{array}\right)\left(\begin{array}{l}
\phi_{1} \\
\phi_{2} \\
\phi_{3}
\end{array}\right)
$$




$$
\left(\begin{array}{l}
\phi_{1} \\
\phi_{2} \\
\phi_{3}
\end{array}\right)_{y}=\left(\begin{array}{ccc}
0 & \mathcal{H} / \lambda & 0 \\
0 & 0 & 1 /\left(\lambda \mathcal{H}^{2}\right) \\
\mathcal{H} / \lambda & 0 & 0
\end{array}\right)\left(\begin{array}{l}
\phi_{1} \\
\phi_{2} \\
\phi_{3}
\end{array}\right)
$$

It is worth pointing out that there exists an alternative bilinearization of the Tziteica equation (1), as a coupled system of $2+1$ dimensional Hirota forms involving only one tau function [18]:

$$
\left\{\begin{array}{c}
\left(D_{x}^{3} D_{y}-D_{y} D_{t}-3 D_{x}^{2}\right) \tau \cdot \tau=0, \\
\left(D_{x}^{3} D_{y}^{3}-D_{y}^{3} D_{t}-9 D_{x}^{2} D_{y}^{2}+18 D_{x} D_{y}\right) \tau \cdot \tau=0, \\
\mathcal{H}=1-2(\log \tau)_{x y} .
\end{array}\right.
$$

In fact, it can be shown that the tau function appearing in (16) is nothing but the Pfaffian tau function $\tau_{\mathrm{Pf}}$ associated with $\tau_{0}$ in (10): $\tau_{0}=\tau^{2}$. Furthermore, these Pfaffians can be shown to be independent of the auxiliary (independent) variable $t$ in (16), a variable that can be thought of as the time variable associated with the third order flow of the BKP hierarchy. This $t$-independence is of course consistent with the fact, that when embedded into an extended BKP hierarchy that also contains compatible negative time flows, a period 3-reduced B-type Toda lattice should really be part of the 3-reduction of that hierarchy.

2. The coupled KP hierarchy and its extension. The system of Hirota equations

$$
\left\{\begin{array}{l}
{\left[D_{1}^{4}-4 D_{1} D_{3}+3 D_{2}^{2}\right] \tau \cdot \tau=24 \bar{\sigma} \sigma} \\
{\left[D_{1}^{3}+2 D_{3}+3 D_{1} D_{2}\right] \sigma \cdot \tau=0} \\
{\left[D_{1}^{3}+2 D_{3}-3 D_{1} D_{2}\right] \bar{\sigma} \cdot \tau=0}
\end{array}\right.
$$

(where $\tau, \sigma, \bar{\sigma}$ depend on the variables $x_{1}, x_{2}, x_{3}$ and where $D_{\ell}$ denotes the Hirota operator w.r.t. $\left.x_{\ell}: D_{\ell}:=D_{x_{\ell}}\right)$ was originally introduced as a coupling of the Kadomtsev-Petviashvili (KP) equation and a higher order Davey-Stewartson equation, allowing for at least 4-soliton interactions [8]. It was subsequently shown to be integrable in the sense that it allows for $N$-soliton interactions (the soliton being expressible in terms of Pfaffians) and that it is actually part of an infinite hierarchy of equations that all share the same set of Pfaffian solutions [9]. Because of the resemblance of the first equation in (18) to the bilinear KP equation - it is of the form ' $\mathrm{KP}+$ source term' - the system (18) is often referred to as the coupled KP equation (in Hirota form). It can be written as a set of $2+1$ dimensional evolution equations, through the dependent variable transformation

$$
\begin{gathered}
u=2(\log \tau)_{2 x_{1}}, \quad \bar{v}=\frac{\bar{\sigma}}{\tau}, \quad v=\frac{\sigma}{\tau}, \\
\left\{\begin{array}{l}
\left(4 u_{x_{3}}-u_{3 x_{1}}-12 u u_{x_{1}}\right)_{x_{1}}-3 u_{2 x_{2}}+12(v \bar{v})_{2 x_{1}}=0 \\
2 \bar{v}_{x_{3}}-3 \bar{v}_{x_{1} x_{2}}+\bar{v}_{3 x_{1}}+6 u \bar{v}_{x_{1}}-6 \bar{v} \int u_{x_{2}} d x_{1}=0, \\
2 v_{x_{3}}+3 v_{x_{1} x_{2}}+v_{3 x_{1}}+6 u v_{x_{1}}+6 v \int u_{x_{2}} d x_{1}=0 .
\end{array}\right.
\end{gathered}
$$


Recently there has been renewed interest in the above system of equations and its associated hierarchy, mainly in connection with the study of matrix integrals [15] and orthogonal polynomials [1, 2], but also because of the remarkable interaction properties of its soliton solutions [10]. It is known that the coupled KP hierarchy is connected to an infinite rank Kac-Moody algebra of D-type [14, 16]; more precisely, it can be shown $[13,17]$ that the coupled KP hierarchy is associated to an algebra (denoted as $D_{\infty}^{\prime}$ ) isomorphic to the usual $D_{\infty}$ algebra. This algebra can be defined in terms of fermion operators $\psi_{i}, \psi_{j}^{*}(i, j \in \mathbb{Z})$ (cf. [13] for details on the algebra of free fermions, $D_{\infty}, D_{\infty}^{\prime}$ and related integrable systems):

$$
\begin{gathered}
{\left[\psi_{i}, \psi_{j}^{*}\right]_{+}=\delta_{i j}, \quad\left[\psi_{i}, \psi_{j}\right]_{+}=\left[\psi_{i}^{*}, \psi_{j}^{*}\right]_{+}=0,} \\
D_{\infty}^{\prime}:=\left\{\sum_{i, j \in \mathbb{Z}} a_{i, j}: \psi_{i} \psi_{j}^{*}:+\sum_{i, j \in \mathbb{Z}} b_{i, j} \psi_{i} \psi_{j}\right. \\
\left.+\sum_{i, j \in \mathbb{Z}} c_{i, j} \psi_{i}^{*} \psi_{j}^{*}+d \mid \exists N, a_{i, j}=b_{i,-j}=c_{-i, j}=0 \quad \text { if }|i-j|>N\right\} .
\end{gathered}
$$

Extending the construction found in [13], one can define tau functions [24]

$$
\tau_{n, \ell}(\mathbf{x}, \mathbf{y})=\left\langle n+\ell\left|e^{H(\mathbf{x})} e^{\bar{H}(\mathbf{y})} e^{X} e^{-\bar{H}(\mathbf{y})}\right| n-\ell\right\rangle, \quad X \in D_{\infty}^{\prime},
$$

on a 2 -dimensional lattice $(n, \ell) \in \mathbb{Z}^{2}$. In this definition, $|\ell\rangle$ (and $\langle n|$ ) denote the highest weight vectors in the Fock space (and its dual) associated to the algebra of free fermion operators $\psi_{i}$ and $\psi_{j}^{*}$. These tau functions depend on two infinite sets of coordinates $\mathbf{x}=\left(x_{1}, x_{2}, x_{3}, \ldots\right)$ and $\mathbf{y}=\left(y_{1}, y_{2}, y_{3}, \ldots\right)$, the time evolution with respect to which is defined in terms of the Hamiltonians:

$$
H(\mathbf{x})=\sum_{n=1}^{\infty} x_{n} \sum_{j \in \mathbb{Z}} \psi_{j} \psi_{j+n}^{*}, \quad \bar{H}(\mathbf{y})=\sum_{n=1}^{\infty} y_{n} \sum_{j \in \mathbb{Z}} \psi_{j} \psi_{j-n}^{*} .
$$

The tau functions (23) can be shown to satisfy the bilinear identity $\left(\forall n, \ell, n^{\prime}, \ell^{\prime} \in \mathbb{Z}\right.$, $\left.\forall \mathbf{x}, \mathbf{y}, \mathbf{x}^{\prime}, \mathbf{y}^{\prime}\right)$

$$
\begin{aligned}
\oint_{\mathcal{C}} d k & {\left[V_{n, \ell}(\mathbf{x}, \mathbf{y}) V_{n^{\prime}, \ell^{\prime}}^{*}\left(\mathbf{x}^{\prime}, \mathbf{y}^{\prime}\right) e^{\xi\left(\mathbf{x}-\mathbf{x}^{\prime}, k\right)+\xi\left(\mathbf{y}-\mathbf{y}^{\prime}, \frac{1}{k}\right)}\right] } \\
+ & \oint_{\mathcal{C}} d k\left[V_{n+1, \ell+1}^{*}(\mathbf{x}, \mathbf{y}) V_{n^{\prime}-1, \ell^{\prime}-1}\left(\mathbf{x}^{\prime}, \mathbf{y}^{\prime}\right) e^{-\xi\left(\mathbf{x}-\mathbf{x}^{\prime}, k\right)-\xi\left(\mathbf{y}-\mathbf{y}^{\prime}, \frac{1}{k}\right)}\right]=0,
\end{aligned}
$$

where $\xi(\mathbf{x}, k):=\sum_{n=1}^{\infty} x_{n} k^{n}$ and where $V_{n, \ell}(\mathbf{x}, \mathbf{y})$ and $V_{n, \ell}^{*}(\mathbf{x}, \mathbf{y})$ are defined through their formal expansions at the singular points $k=\infty$ and $k=0$ of the exponentials that appear in the integrands of (25):

$$
\begin{gathered}
V_{n, \ell}(\mathbf{x}, \mathbf{y})=\left\{\begin{array}{ll}
k^{n+\ell} \tau_{n, \ell}\left(\mathbf{x}-\boldsymbol{\varepsilon}\left[k^{-1}\right], \mathbf{y}\right), & k \approx \infty \\
k^{n-\ell} \tau_{n+1, \ell}(\mathbf{x}, \mathbf{y}-\boldsymbol{\varepsilon}[k]), & k \approx 0
\end{array},\right. \\
V_{n, \ell}^{*}(\mathbf{x}, \mathbf{y})=\left\{\begin{array}{ll}
k^{-n-\ell} \tau_{n, \ell}\left(\mathbf{x}+\boldsymbol{\varepsilon}\left[k^{-1}\right], \mathbf{y}\right), & k \approx \infty \\
k^{\ell-n} \tau_{n-1, \ell}(\mathbf{x}, \mathbf{y}+\boldsymbol{\varepsilon}[k]), & k \approx 0
\end{array},\right.
\end{gathered}
$$


with $\varepsilon[k]=\left(k, \frac{k^{2}}{2}, \frac{k^{3}}{3}, \ldots\right)$. The contour $\mathcal{C}$ is such that both $k=\infty$ and $k=0$ are contained within it and one can think of the integrals in (25) as the sum of the residues of the integrands at those two points.

The coupled KP hierarchy is obtained from the bilinear identity (25) restricted to a diagonal on the $(n, \ell)$ lattice and taken at the point $\mathbf{y}=\mathbf{y}^{\prime}=\mathbf{0}$. More precisely, it is the set of equations for tau functions $\tau_{\ell}(\mathbf{x})$ :

$$
\begin{gathered}
\tau_{\ell}(\mathbf{x}):=\tau_{\ell / 2, \ell / 2}(\mathbf{x}, \mathbf{y}=\mathbf{0}) \quad \text { if } \ell \text { is even, } \\
\text { or } \tau_{(\ell+1) / 2,(\ell-1) / 2}(\mathbf{x}, \mathbf{y}=\mathbf{0}) \quad \text { if } \ell \text { is odd. }
\end{gathered}
$$

In particular, comparing with the system (18), one has that

$$
\tau \equiv \tau_{\ell}(\mathbf{x}), \quad \bar{\sigma} \equiv \tau_{\ell+2}(\mathbf{x}), \quad \sigma \equiv \tau_{\ell-2}(\mathbf{x}),
$$

for some $\ell \in \mathbb{Z}$ and for a particular $X \in D_{\infty}^{\prime}$.

However, allowing the tau functions $\tau_{n, \ell}(\mathbf{x}, \mathbf{y})$ to evolve in the "negative weight" variables $\mathbf{y}$ as well, the bilinear identity (25) yields the following coupled set of lattice equations, at lowest weight ( $x=x_{1}, y=y_{1}$ for simplicity):

$$
\left\{\begin{array}{l}
\frac{1}{2} D_{x} D_{y} \tau_{n, \ell} \cdot \tau_{n, \ell}=\left(\tau_{n, \ell}^{2}-\tau_{n+1, \ell} \tau_{n-1, \ell}\right)+\tau_{n, \ell+1} \tau_{n, \ell-1}, \\
D_{y} \tau_{n+1, \ell+1} \cdot \tau_{n, \ell}+D_{x} \tau_{n+1, \ell} \cdot \tau_{n, \ell+1}=0 .
\end{array} \quad(n \in \mathbb{Z}, l \in \mathbb{Z})\right.
$$

This system of Hirota equations can be seen to determine an integrable lattice, defined on an infinite strip $\mathcal{D}_{(n, \ell)}$ of width 3 (in the $\ell$-direction) centered around an arbitrary point of the tau function lattice $(n, \ell) \in \mathbb{Z}^{2}$ :

$$
\mathcal{D}_{(n, \ell)}=\left\{\left(n+\delta_{n}, \ell+\delta_{\ell}\right) \in \mathbb{Z}^{2} \mid \delta_{n} \in \mathbb{Z}, \delta_{\ell}=0, \pm 1\right\} .
$$

Indeed, defining new dependent variables $\theta_{n}, \bar{v}_{n}$ and $v_{n}$ in terms of the tau functions in $\mathcal{D}_{(n, \ell)}$

$$
\begin{gathered}
\theta_{n}(x, y ; \ell):=\log \frac{\tau_{n, \ell}^{2}}{\tau_{n+1, \ell} \tau_{n-1, \ell}}, \\
\bar{v}_{n}(x, y ; \ell):=\frac{\tau_{n+1, \ell+1}}{\tau_{n, \ell}}, \quad v_{n}(x, y ; \ell):=\frac{\tau_{n-1, \ell-1}}{\tau_{n, \ell}},
\end{gathered}
$$

(31) gives rise to a system of coupled lattice equations [24] $(\forall n \in \mathbb{Z})$

$$
\left\{\begin{array}{l}
\left(\theta_{n}\right)_{x y}=2\left(v_{n+1} \bar{v}_{n-1}-1\right) e^{-\theta_{n}}-\left(v_{n+2} \bar{v}_{n}-1\right) e^{-\theta_{n+1}}-\left(v_{n} \bar{v}_{n-2}-1\right) e^{-\theta_{n-1}}, \\
\left(\bar{v}_{n}\right)_{y}=\left(\bar{v}_{n-1}\right)_{x} e^{-\theta_{n}}+\left(\theta_{n}+2 \Delta^{-1}\left[\theta_{n}\right]\right)_{x} \bar{v}_{n-1} e^{-\theta_{n}} \\
\left(v_{n}\right)_{y}=\left(v_{n+1}\right)_{x} e^{-\theta_{n}}-\left(\theta_{n}+2 \Delta^{-1}\left[\theta_{n}\right]\right)_{x} v_{n+1} e^{-\theta_{n}}
\end{array}\right.
$$

(where $\Delta^{-1}\left[\theta_{n}\right]:=-\sum_{j=0}^{+\infty} \theta_{n+j}$ ) that arises as the compatibility condition of the linear system

$$
\begin{aligned}
& \left(\begin{array}{l}
\psi_{n} \\
\chi_{n}
\end{array}\right)_{x}=\left(\begin{array}{cc}
\left(\eta_{n}\right)_{x}+\mathcal{S} & \bar{v}_{n} \mathcal{S} \\
-v_{n} \mathcal{S}^{-1} & -\left(\eta_{n-1}\right)_{x}-\mathcal{S}^{-1}
\end{array}\right)\left(\begin{array}{l}
\psi_{n} \\
\chi_{n}
\end{array}\right), \\
& \left(\begin{array}{l}
\psi_{n} \\
\chi_{n}
\end{array}\right)_{y}=\left(\begin{array}{cc}
e^{-\theta_{n}} \mathcal{S}^{-1} & \bar{v}_{n-1} e^{-\theta_{n}} \mathcal{S} \\
-v_{n+1} e^{-\theta_{n}} \mathcal{S}^{-1} & -e^{-\theta_{n}} \mathcal{S}
\end{array}\right)\left(\begin{array}{l}
\psi_{n} \\
\chi_{n}
\end{array}\right) .
\end{aligned}
$$


Here, the auxiliary variable $\eta_{n}(x, y ; \ell)$ is related to $\theta_{n}$ by

$$
\eta_{n}(x, y ; \ell)=\log \frac{\tau_{n+1, \ell}}{\tau_{n, \ell}}, \quad \theta_{n}=\eta_{n-1}-\eta_{n},
$$

and the symbol $\mathcal{S}$ stands for a shift operator acting on the variable $n, \mathcal{S}: f(n) \rightarrow$ $f(n+1)$.

The structure of the system (35) is remarkably similar to that of the coupled KP equations (20): it consists of a Toda-like equation (instead of a KP-like equation) coupled to two linear equations, each containing an "integrated" term. The author will resist the temptation of referring to the lattice (35) as a coupled Toda lattice; yet, it is important to point out that this is exactly what (35) is, and actually in more ways than one. Going back to the first equation in the bilinear system (31) (the one that gives rise to the equation for $\theta_{n}$ in (35)) one notices that it describes the coupling of a standard 2DToda lattice in the $n$ direction to a so called molecule Toda lattice (i.e., a non-periodic finite Toda lattice, governed by a Hirota equation of the type $\left.\frac{1}{2} D_{x} D_{y} \tau_{\ell} \cdot \tau_{\ell}=\tau_{\ell+1} \tau_{\ell-1}\right)$ in the $\ell$-direction. One could argue that this interpretation of the two directions in the lattice is just the result of the suggestive grouping of the terms in the right-hand side of (31) and that one could just as easily claim the converse. However, it can be seen from the definition of the tau functions (23) that the former interpretation is actually correct. A discussion of the solutions of (31) would lead us too far, but typically one can say that they are described by Pfaffians of the same size running along the $n$-direction and with decreasing size for increasing $|\ell|$ (i.e., with maximal size at $\ell=0$ and vanishing at a certain distance from $\ell=0$ ). Finally, note that although the system (31) (or (35)) is related to an algebra isomorphic to $D_{\infty}$, it differs completely from the usual D-type Toda lattice which is known to be related to certain non-commutative Toda lattices $[20,21]$.

3. Reductions of the coupled KP lattice. Just as was the case for the Toda lattice, the lattice (35) contains various interesting (integrable) sub-systems. Below, the analogue of periodic reductions for this lattice and of a reduction by folding between two lattice points will be described. The case of a reduction by folding on a lattice point will be described at a future occasion.

3.1. Periodic reductions. A periodic reduction in the $n$-direction of the tau function lattice (31), $\forall \mathbf{x}, \mathbf{y}, n, \ell: \tau_{n+p, \ell}=\tau_{n, \ell}$, can be shown to correspond to the following restriction of $D_{\infty}^{\prime}$ to a subalgebra $\mathcal{P}^{\prime}$, by means of the automorphism $\iota_{p}$ :

$$
\begin{gathered}
\iota_{p}\left(\psi_{j}\right)=\psi_{j-p}, \quad \iota_{p}\left(\psi_{j}^{*}\right)=\psi_{j-p}^{*}, \\
\mathcal{P}^{\prime}=\left\{X \in D_{\infty}^{\prime} \mid\left[\operatorname{ad}_{X}, \iota_{p}\right]_{-}=0\right\} \\
=\left\{X \in D_{\infty}^{\prime} \mid a_{i+p, j+p}=a_{i, j}, b_{i+p, j+p}=b_{i, j}, c_{i+p, j+p}=c_{i, j}\right\} .
\end{gathered}
$$

An immediate consequence of the periodicity of the lattice in the $n$-direction is that $\sum_{n=0}^{p-1} \theta_{n}=0$ and hence that the $\Delta^{-1}$ term in (35) can be resolved. However, contrary to what happens for the usual Toda lattice, periodicity does not imply that the tau 
functions generated from $X \in \mathcal{P}^{\prime}$ become independent of certain higher order time flows. In particular $\tau_{n+p, \ell}=\tau_{n, \ell}$ does not imply $\tau_{x_{p}}=\tau_{y_{p}}=0$. The reader is referred to [24] for an example of a reduction at $p=2$, resulting in a coupling of the sinh-Gordon equation to a set of linear equations.

3.2. "Twisted" folding between lattice points. Another restriction of $D_{\infty}^{\prime}$ that leads to interesting integrable equations is obtained by means of the automorphism $\sigma_{0}:$

$$
\begin{gathered}
\sigma_{0}\left(\psi_{j}\right)=(-)^{j} \psi_{-j}^{*}, \quad \sigma_{0}\left(\psi_{j}^{*}\right)=(-)^{j} \psi_{-j}, \\
\left\{X \in D_{\infty}^{\prime} \mid \sigma_{0}[X]=X\right\}=\left\{X \in D_{\infty}^{\prime} \mid a_{i, j}=(-)^{1+i+j} a_{-j,-i}, b_{i, j}=(-)^{i+j} c_{-i,-j}\right\} .
\end{gathered}
$$

In this case, just as for B-type reductions of the usual KP hierarchy, the evolution of the corresponding tau functions can only be defined with respect to the even weight flows. We therefore introduce a restricted set of coordinates $(\overline{\mathbf{x}}, \overline{\mathbf{y}})=\left(\left.\mathbf{x}\right|_{x_{\text {even }}=0},\left.\mathbf{y}\right|_{y_{\text {even }}=0}\right)$ with respect to which we define $\tau(\overline{\mathbf{x}}, \overline{\mathbf{y}})$ as in (23), for elements of the algebra (42). It can then be shown that such tau functions satisfy

$$
\tau_{n, \ell}(\overline{\mathbf{x}}, \overline{\mathbf{y}})=(-)^{\ell} \tau_{1-n,-\ell}(\overline{\mathbf{x}}, \overline{\mathbf{y}})
$$

This constraint corresponds to folding the tau function lattice (31) in between the lines $n=1$ and $n=0$ but with an extra exchange of the indices $\ell$ and $-\ell$ added. Therefore, taken for a general point $(n, \ell) \in \mathbb{Z}^{2}$ this constraint cannot be satisfied within a single strip $\mathcal{D}_{(n, \ell)}$, except for the strip $\mathcal{D}_{(n, 0)}$, where it corresponds to folding the strip between $n=0$ and $n=1$ while twisting it such that $\ell \rightarrow-\ell$. Hence, the above constraint will only result in a reduction of the lattice (35) when one restricts oneself to a system defined in terms of tau functions in $\mathcal{D}_{(n, 0)}$, i.e. in a strip of width 3 centered around $\ell=0$.

3.3. A coupled Tzitzeica equation. Consider a twisted folding in $\mathcal{D}_{(n, 0)}$, as explained above, combined with a periodic reduction at $p=3$. Just as was the case for periodic reductions of the B-type Toda lattice, the combination of these two reductions induces special relations between the tau functions

$$
\begin{aligned}
& \tau_{-2, \ell}=\tau_{1, \ell}=(-)^{\ell} \tau_{0,-\ell}, \\
& \tau_{-1,1}=\tau_{2,1}=-\tau_{-1,-1},
\end{aligned}
$$

which can then be used to obtain the following system of Hirota equations from (31), defined on only 5 points $\{(0,-1),(0,0),(0,1),(-1,-1),(-1,0)\}$ in $\mathcal{D}_{(n, 0)}$ :

$$
\left\{\begin{array}{l}
\frac{1}{2} D_{x} D_{y} \tau_{0,0} \cdot \tau_{0,0}=\left(\tau_{0,0}^{2}-\tau_{0,0} \tau_{-1,0}\right)+\tau_{0,1} \tau_{0,-1}, \\
\frac{1}{2} D_{x} D_{y} \tau_{-1,0} \cdot \tau_{-1,0}=\left(\tau_{-1,0}^{2}-\tau_{0,0}^{2}\right)-\tau_{-1,-1}^{2}, \\
D_{y} \tau_{0,-1} \cdot \tau_{0,0}-D_{x} \tau_{0,0} \cdot \tau_{0,1}=0, \\
D_{y} \tau_{0,1} \cdot \tau_{-1,0}-D_{x} \tau_{0,0} \cdot \tau_{-1,-1}=0 \\
D_{y} \tau_{0,0} \cdot \tau_{-1,-1}+D_{x} \tau_{0,-1} \cdot \tau_{-1,0}=0 .
\end{array}\right.
$$


This set of equations describes an integrable reduction of (35). Introducing variables that differ slightly from those defined in (33) and (34),

$$
\begin{gathered}
h=\frac{\tau_{-1,0}}{\tau_{0,0}}, \quad v=-\frac{\tau_{-1,-1}}{\tau_{0,0}}, \\
\omega=-\frac{\tau_{0,-1}}{\tau_{0,0}}, \quad \bar{\omega}=\frac{\tau_{0,1}}{\tau_{0,0}},
\end{gathered}
$$

one obtains an integrable system that generalizes the Tzitzeica equation to a coupled system of equations

$$
\left\{\begin{array}{l}
(\log h)_{x y}=h-\frac{1+v^{2}}{h^{2}}+\omega \bar{\omega} \\
v_{x}=\bar{\omega}_{y} h-\bar{\omega} h_{y} \\
v_{y}=\omega_{x} h-\omega h_{x} \\
\omega_{y}=\bar{\omega}_{x} .
\end{array}\right.
$$

Performing the above reduction on the linear system $(36,37)$ in the same vein as for the Toda lattice, one easily obtains a Lax pair for this coupled Tzitzeica equation:

$$
\begin{aligned}
& \left(\begin{array}{l}
\psi_{0} \\
\psi_{1} \\
\psi_{2} \\
\chi_{0} \\
\chi_{1} \\
\chi_{2}
\end{array}\right)_{x}=\left(\begin{array}{cccccc}
0 & 0 & \lambda & 0 & 0 & \omega / \lambda \\
\lambda & -h_{x} / h & 0 & \bar{\omega} /(\lambda h) & 0 & 0 \\
0 & \lambda & h_{x} / h & 0 & v / \lambda & 0 \\
0 & v / \lambda & 0 & h_{x} / h & -1 / \lambda & 0 \\
0 & 0 & \lambda \bar{\omega} / h & 0 & -h_{x} / h & -1 / \lambda \\
\lambda \omega & 0 & 0 & -1 / \lambda & 0 & 0
\end{array}\right)\left(\begin{array}{l}
\psi_{0} \\
\psi_{1} \\
\psi_{2} \\
\chi_{0} \\
\chi_{1} \\
\chi_{2}
\end{array}\right), \\
& \left(\begin{array}{l}
\psi_{0} \\
\psi_{1} \\
\psi_{2} \\
\chi_{0} \\
\chi_{1} \\
\chi_{2}
\end{array}\right)_{y}=\left(\begin{array}{cccccc}
0 & h / \lambda & 0 & 0 & 0 & \bar{\omega} / \lambda \\
0 & 0 & 1 /\left(\lambda h^{2}\right) & \nu /\left(\lambda h^{2}\right) & 0 & 0 \\
h / \lambda & 0 & 0 & 0 & \omega h / \lambda & 0 \\
0 & \lambda \omega h & 0 & 0 & 0 & -\lambda h \\
0 & 0 & \lambda v / h^{2} & -\lambda / h^{2} & 0 & 0 \\
\lambda \bar{\omega} & 0 & 0 & 0 & -\lambda h & 0
\end{array}\right)\left(\begin{array}{l}
\psi_{0} \\
\psi_{1} \\
\psi_{2} \\
\chi_{0} \\
\chi_{1} \\
\chi_{2}
\end{array}\right) .
\end{aligned}
$$

Clearly, when $\omega=\bar{\omega}=v=0$, this Lax pair reduces to two (disjoint) copies of the Lax pair for the classical Tzitzeica equation (1).

4. Conclusions. The extension of the coupled KP hierarchy to coupled Todalike systems as presented above, appears to contain various interesting integrable systems. One of them, obtained by a twisted folding of the two dimensional lattice on which the extended coupled KP tau functions are defined, corresponds to a coupling of the Tzitzeica equation to a set of linear equations. This system resembles the Gauss-Mainardi-Codazzi equations for hyperbolic surfaces in centroaffine geometry, expressed in terms of asymptotic coordinates (cf. system (19) in [22]). Indeed, introducing scaled coordinates and variables $x=i X, y=i Y, h(x, y)=$ $-H(X, Y), \omega=i R_{X}, \bar{\omega}=i R_{Y}$ and representing $v$ as $v=\frac{A-1}{i}=\frac{B+1}{i}$, the coupled

Tzitzeica equation (49) is transformed into

$$
\begin{gathered}
(\log H)_{X Y}=H-\frac{A B}{H^{2}}+R_{X} R_{Y}, \\
A_{Y}=R_{2 X} H-R_{X} H_{X}, \quad B_{X}=R_{2 Y} H-R_{Y} H_{Y} .
\end{gathered}
$$


In general, i.e., relaxing the constraint $B=A-2$ implicit in the above derivation, this system is underdetermined, but several integrable and geometrically interesting subcases are known, besides the above case with $B=A-2$. Most notably, for constant curvature $R$ (52) reduces to the Tzitzeica equation (1) (as $A$ and $B$ can be scaled to unity). In [22] another geometrically interesting case is investigated : that of generalized affine spheres for which $R$ is linear in the coordinates $X$ and $Y$. For the present case, derived from the integrable system (49), there is no clear-cut geometric interpretation that comes to mind, not in the least because of the relation $B=A-2$ it requires. The nature of the Lax pair $(50,51)$ however hints at a possible connection with certain generalizations of Demoulin surfaces $[4,5]$, a connection which remains to be investigated.

The description of explicit solutions for the coupled Tzitzeica equation and of its integrable discretizations, as well as the investigation of other integrable subcases of the lattice (35) are also the subject of future research.

ACKNOWLEDGEMENTS. This work was partially supported by a Grant-In-Aid from the Japan Society for the Promotion of Science (JSPS).

\section{REFERENCES}

1. M. Adler, E. Horozov and P. van Moerbeke, The Pfaff lattice and skew-orthogonal polynomials, Int. Math. Res. Notices 11 (1999), 569-588.

2. M. Adler, T. Shiota and P. van Moerbeke, Pfaff $\tau$-functions, Math. Ann. 322 (2002), 423-476.

3. R. K. Dodd and R. K. Bullough, Polynomial conserved densities for the sine-Gordon equations, Proc. Roy. Soc. London A 352 (1977), 481-503.

4. E. V. Ferapontov, Integrable systems in projective differential geometry, Kyushu J. Math. 54 (2000), 183-215.

5. E. V. Ferapontov and W. K. Schief, Surfaces of Demoulin: differential geometry, Bäcklund transformation and integrability, J. Geom. Phys. 30 (1999), 343-363.

6. A. P. Fordy and J. Gibbons, Integrable nonlinear Klein-Gordon equations and Toda lattices, Comm. Math. Phys. 77 (1980), 21-30.

7. M. Guest, R. Miyaoka and Y. Ohnita (Eds.), Differential geometry and integrable systems, Contemporary Mathematics 308 (2002).

8. J. Hietarinta, Hirota's bilinear method and partial integrability, in Partially integrable evolution equations in Physics (Eds. R. Conte and N. Boccara) (Kluwer Acad. Publ., Dordrecht, 1990), 459-478.

9. R. Hirota and Y. Ohta, Hierarchies of coupled soliton equations. I, J. Phys. Soc. Japan. 60 (1991), 798-809.

10. S. Isojima, R. Willox and J. Satsuma, Spider-web solutions of the coupled KP equation, J. Phys. A 36 (2003), 9533-9552.

11. A. R. Its, Liouville's theorem and the method of the inverse problem, J. Sov. Math. 31 (1985), 3330-3338.

12. A. V. Jiber and A. B. Shabat, Klein-Gordon equations with a non-trivial group, Sov. Phys. Dokl. 24 (1979), 607-609.

13. M. Jimbo and T. Miwa, Solitons and infinite dimensional Lie algebras, Publ. RIMS 19 (1983), 943-1001.

14. V. Kac and J. van de Leur, The geometry of spinors and the multicomponent BKP and DKP hierarchies, CRM Proceedings and Lecture Notes 14 (1998), 159202.

15. S. Kakei, Orthogonal and symplectic matrix integrals and coupled KP hierarchy, J. Phys. Soc. Japan. 68 (1999), 2875-2877. 
16. S. Kakei, The algebraic structure of the coupled KP hierarchy, Proceedings of the Research Institute for Applied Mechanics, Kyushu University, 11ME-S4 (2000), 178-182. [In Japanese]

17. S. Kakei, Symmetry, discretization and ultra-discretization of the coupled KP hierarchy, RIMS Kokyuroku 1221 (2001), 199-208. [In Japanese]

18. F. Lambert, I. Loris, J. Springael and R. Willox, On the Hirota representation of soliton equations with one tau-function, J. Phys. Soc. Japan. 70 (2001), 605-608.

19. A. V. Mikhailov, Integrability of a two-dimensional generalization of the Toda chain, JETP Lett. 30 (1979), $414-418$.

20. A. V. Mikhailov, The reduction problem and the inverse scattering method, Physica D 3 (1981), 73-117.

21. J. J. C. Nimmo and R. Willox, Darboux transformations for the two-dimensional Toda system, Proc. Roy. Soc. London A 453 (1997), 2497-2525.

22. W. K. Schief, Hyperbolic surfaces in centro-affine geometry-Integrability and discretization, Chaos, Solitons and Fractals 11 (2000), 97-106.

23. G. Tzitzeica, Sur une nouvelle classe de surfaces, C. R. Acad. Sci. 150 (1910), 955-956.

24. R. Willox, On a coupled Toda lattice and its reductions as derived from the coupled KP hierarchy, Proceedings of the Research Institute for Applied Mechanics, Kyushu University, 13ME-S4 (2002), 18-23. [In Japanese] 
